\title{
Transverse-momentum selection rules for ballistic electrons at epitaxial metal/GaAs(001) interfaces
}

\author{
S. Guézo, P. Turban, S. Di Matteo, P. Schieffer, S. Le Gall, B. Lépine, C. Lallaizon, and G. Jézéquel \\ Equipe de Physique des Surfaces et Interfaces, Institut de Physique de Rennes UMR UR1-CNRS 6251, Université de Rennes 1, \\ F-35042 Rennes Cedex, France
}

(Received 1 December 2009; published 12 February 2010)

\begin{abstract}
We report on ballistic electron-emission spectroscopy on high-quality $\mathrm{Au}(110) / \mathrm{GaAs}(001)$ and $\mathrm{Fe}(001)$ / $\mathrm{GaAs}(001)$ Schottky contacts. For the $\mathrm{Au}(110) / \mathrm{GaAs}(001)$ interface, the ballistic current is characterized by a strong electron injection in the $L$ valley of the GaAs conduction band. This remarkable spectroscopic feature is absent for the $\mathrm{Fe}(001) / \mathrm{GaAs}(001)$ interface. These observations are explained by the different electronic structures in the two metal layers, assuming conservation of the electron transverse momentum at the metal/ semiconductor epitaxial interfaces. Conversely, this comparative study suggests that the technique can be used for the analysis of local electronic states propagating in the metal films.
\end{abstract}

DOI: 10.1103/PhysRevB.81.085319

PACS number(s): 73.40.-c, 73.20.At, 73.23.Ad

Ballistic electron-emission microscopy (BEEM) is an experimental technique measuring the electron current which, injected from the tip of a scanning tunneling microscope (STM) into a thin metallic overlayer, travels through an underlying metal-semiconductor (MS) interface and is finally collected in the semiconductor. ${ }^{1}$ Since its discovery, the main applications of BEEM were focused on the study of Schottky-barrier heights (SBHs) and of electron transport across metal/semiconductor interfaces, with a nanometer lateral resolution. ${ }^{2,3}$ Given the current trend in the development of electronic devices at the nanometer scale, these properties make the BEEM technique a powerful tool for the characterization and the control of MS heterostructures.

The original theoretical description ${ }^{1}$ was based on the two main assumptions: (i) the propagation in the metal layer is that of a free electron with renormalized mass; (ii) both energy and momentum component parallel to the interface $\left(k_{\|}\right)$ are conserved as the electron crosses the MS interface. Yet, the applications of such a model were found to be at odds with the experimental evidence. For example, in the case of $\mathrm{Au} / \mathrm{Si}(100)$ and $\mathrm{Au} / \mathrm{Si}(111)$, from these assumptions it was predicted that electrons crossed the $\mathrm{Au} / \mathrm{Si}$ interface almost perpendicularly to it. Under these conditions the conservation of $k_{\|}$would imply a much higher current for the Au/ $\mathrm{Si}(100)$ interface than for the $\mathrm{Au} / \mathrm{Si}(111)$, as the (100) $\mathrm{Si}$ surface allows a direct injection of electrons into the Si available states at the $\bar{\Gamma}$ point, contrary to the (111) Si surface, where no empty density of states is available at the $\bar{\Gamma}$ point. However, the experimental evidence is opposite: the BEEM current for the $\mathrm{Au} / \mathrm{Si}(111)$ interface is about $50 \%$ higher than for the $\mathrm{Au} / \mathrm{Si}(100)$ interface. $^{4}$

In order to explain such a behavior two different modifications of the original theoretical framework were suggested in the literature, based on the lifting of either (i) or (ii). Hypothesis (ii) was abandoned, e.g., in Refs. 5 and 6, where it is supposed that the presence of scattering centers at the interface, preventing the conservation of $k_{\|}$, also provides the finite amount of $k_{\|}$required to reach the available states at the (111) Si surface. On the contrary, $k_{\|}$is conserved at the interface in the approach proposed by García-Vidal et al., ${ }^{7}$ where assumption (i) is lifted. They showed that just five layers $(\sim 1.2 \mathrm{~nm})$ of gold force the electrons to feel the effects of the three-dimensional (3D) crystal band structure, which implies a lack of perpendicular propagation when gold is stacked along the (111) direction. It is therefore the 3D crystal structure of gold, requiring that electrons propagate at angles considerably away from the (111) direction, that provides the transverse momentum necessary to allow the transport through the $\mathrm{Au} / \mathrm{Si}(111)$ interface. It should be noticed that the two assumptions are not necessarily in opposition [one could suppose to lift both (i) and (ii) in order to describe the BEEM data with both band-structure effects and interface scattering centers], and yet there is a basic conceptual difference between them. In fact, the hypothesis of scattering centers at the interface strongly depends on the quality of the interface (it seems reasonable for a nonepitaxial interface but it appears questionable for an ideal epitaxial interface), whereas the idea of considering the electrons in the metal as Bloch electrons is a general property of the metallic film. Therefore one might suspect that, by properly preparing MS interfaces, it may become possible to avoid eventual disturbing scattering events and to demonstrate the main thesis of Ref. 7. Indeed, very recently the authors of Ref. 8 provided convincing experimental evidence in this respect for the epitaxial system $\mathrm{Bi}(111) / \mathrm{Si}(100)$ and $\mathrm{Bi}(111) / \mathrm{Si}(111)$.

In such a context, the aim of our work is twofold: on one side we demonstrate that the main thesis of Ref. 7 is valid also in a system, $\mathrm{Au} / \mathrm{GaAs}$, that in the past was considered as the prototypical nonepitaxial interface. ${ }^{3}$ In order to do this, we shall employ a high-quality epitaxial $\mathrm{Au}(110) / \mathrm{GaAs}(001)$ interface, as detailed below. Second and more important, we switch the point of view usually adopted in the BEEM literature on this subject and demonstrate the fundamental conceptual character of the idea advanced in Ref. 7 by keeping fixed the substrate (GaAs) and by changing the metallic thin film ( $\mathrm{Au}$ and $\mathrm{Fe}$ ), instead of fixing the metallic system and changing the substrate orientation as done up to now. This allows introducing a comparative analysis on different systems to use BEEM for local investigation of propagating electronic states in the metal film, even buried ones, when the electronic states of the semiconducting substrate are known. In order to do that it is mandatory to use epitaxial 


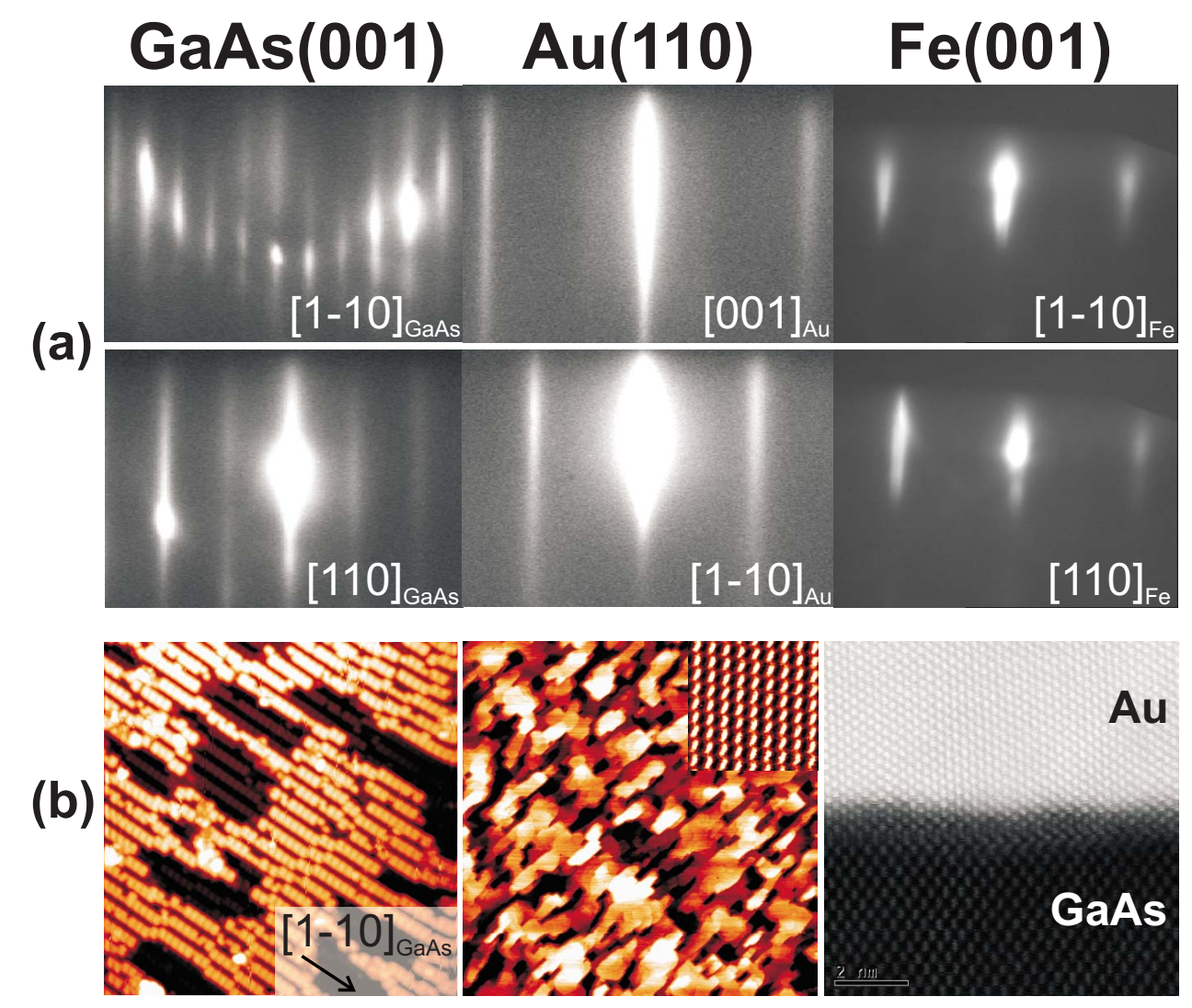

FIG. 1. (Color online) (a) RHEED patterns observed on the As-rich GaAs (001)-(2×4) surface (left) before either the 5-nm-thick Au layer deposition (in the middle) or the 3-nm-thick Fe layer deposition (right). Electron beam is parallel to the direction indicated in each right corner of RHEED pattern. (b) STM topography images of As-rich GaAs(001)- $(2 \times 4)\left(37 \times 37 \mathrm{~nm}^{2}\right)$ and $\mathrm{Au}(110)\left(500 \times 500 \mathrm{~nm}^{2}\right)$. The color scale varies from 0 to $0.42 \mathrm{~nm}$ and $1.76 \mathrm{~nm}$, respectively. The inset is a STM image of a $4.5 \times 4.5 \mathrm{~nm}^{2} \mathrm{Au}(110)$ surface with atomic resolution. The right image is a HAADF-STEM of $\mathrm{Au}(110) / \mathrm{GaAs}(001)$ interface obtained along the $(110)_{\mathrm{GaAs}}$ cross-section plane.

interfaces of very high quality to ensure $k_{\|}$conservation.

The epitaxial Schottky contacts were grown by molecularbeam epitaxy. A $1.5 \mu \mathrm{m}$ Si $n$-doped $\left(4 \times 10^{16} \mathrm{~cm}^{-3}\right) \mathrm{GaAs}$ buffer layer was grown on a $n^{+}-\mathrm{GaAs}(001)$ commercial substrate. Thin $\mathrm{Au}$ or Fe metallic films (thickness 5 and $3 \mathrm{~nm}$, respectively) were evaporated at room temperature (RT) on the GaAs As $(2 \times 4)$-terminated surface from Knudsen cells. The SBHs of the formed contacts were determined by classical current and capacitance versus voltage macroscopic measurements. At RT, the obtained SBH are $\Phi_{\mathrm{Au}}$ $=0.87 \pm 0.04 \mathrm{eV}$ and $\Phi_{\mathrm{Fe}}=0.73 \pm 0.04 \mathrm{eV}$, without corrections of the image potential. The corresponding ideality factor values are $n_{\mathrm{Au}}=1.02$ and $n_{\mathrm{Fe}}=1.03$, typical of highquality Schottky junctions. Electrochemically etched W STM tips were cleaned in situ by thermal heating before the BEEM experiments. All STM/BEEM experiments were performed at RT in the constant-current mode of operation. Details on the experimental setup can be found elsewhere. ${ }^{9}$

Growth and structural properties of $\mathrm{Au} / \mathrm{GaAs}$ and $\mathrm{Fe} /$ GaAs epitaxial systems were first intensively characterized by reflection high-energy electron diffraction (RHEED), STM, and transmission electron microscopy (TEM), as shown in Fig. 1. Figure 1(a) displays the RHEED patterns recorded on the sample's surface after each growth's step when the electron beam is aligned along the $[1 \overline{1} 0]_{\text {GaAs }}$ and $[110]_{\text {GaAs }}$ substrate directions. Before metals deposition, the
GaAs RHEED patterns present typical additional streaks related to the well-known GaAs(001) As $(2 \times 4)$-terminated surface. After deposition of a 5-nm-thick Au layer, narrow diffraction streaks indicate an epitaxial growth with a flat surface at the electrons coherence length scale. The presented RHEED patterns are typical of purely single-crystal Au layers: the presence of twins or disoriented grains would have led to additional diffraction streaks which are not observed on the whole samples surface. The in-plane lattice spacing recorded along substrate high-symmetry directions scales with a factor $\sqrt{2}$ and demonstrates a single-domain $\mathrm{Au}(110)$ growth on $\mathrm{GaAs}(001) .{ }^{10}$ The corresponding epitaxial relationship is $\mathrm{Au}(110)[001] \| \mathrm{GaAs}(001)[1 \overline{1} 0]$. Highquality epitaxial growth of $\mathrm{Au}(110) / \mathrm{GaAs}(001)$ is confirmed in real space by STM observations [Fig. 1(b)]. Atomic resolution is obtained on the $\mathrm{As}(2 \times 4)$-reconstructed $\mathrm{GaAs}(001)$ surface and allows the indexation of main surface directions. After $\mathrm{Au}$ deposition, elongated $\mathrm{Au}(110)$ islands (typical width around $50 \mathrm{~nm}$ and length around $85 \mathrm{~nm}$ ) with a topmost flat surface expand parallel to the $[110]_{\mathrm{GaAs}} \mathrm{di}-$ rection. An inset shows an atomically resolved tunnel current image. The $\mathrm{Au}(110)$ surface presents a $c(2 \times 2)$ atomic order due to the segregation of half a monolayer of $\mathrm{Ga}$ atoms from the interface during growth. ${ }^{10}$ Finally, highangle annular dark-field scanning transmission electron micrograph (HAADF-STEM) observation of the sample with 

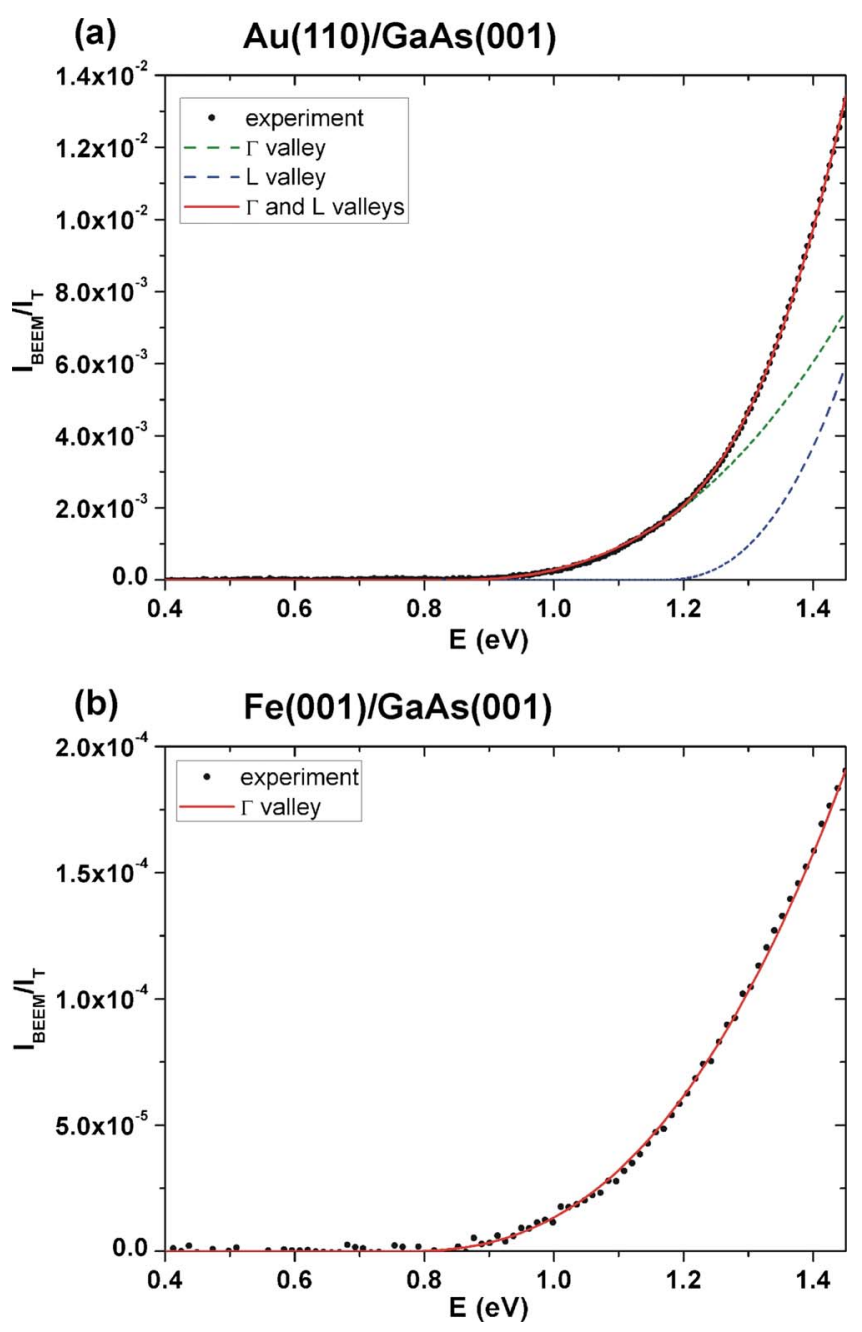

FIG. 2. (Color online) BEEM spectra obtained: (a) on $\mathrm{Au}(110) /$ GaAs(001) sample, average of 6400 spectra (dots), $I_{\mathrm{T}}=10 \mathrm{nA}$. Two GaAs valleys contributions, $\Phi_{\Gamma}$ and $\Phi_{L}$ (following the LP $5 / 2$ power law), are represented by dashed lines and the total fit by a continuous line. (b) On $\mathrm{Fe}(001) / \mathrm{GaAs}(001)$ sample, average of 400 spectra (dots), $I_{\mathrm{T}}=20 \mathrm{nA}$, with one fit contribution $\Phi_{\Gamma}$ (continuous line).

$(110)_{\mathrm{GaAs}}$ cross-section plane demonstrates the abrupt and structurally continuous nature of the $\mathrm{Au}(110) / \mathrm{GaAs}(001)$ interface. For the 3-nm-thick Fe layer growth, the spotty RHEED patterns correspond to a 3D Fe(001) islands growth with the already well-documented epitaxial relationship $\mathrm{Fe}(001)[100] \| \mathrm{GaAs}(001)[100] .^{11}$ Previous high-resolution TEM studies extensively demonstrated structural continuity at the $\mathrm{Fe}(001) / \mathrm{GaAs}(001)$ interface. ${ }^{12}$ Of course, we do not exclude the presence of point defects and dislocations at the metals/GaAs(001) interface. The density of interface defects is expected to be very low in the case of the $\mathrm{Fe}(001) /$ GaAs(001) sample, whose thickness is $3 \mathrm{~nm}$, and is expected to be just slightly relaxed (see Ref. 13), but it is expected to be bigger in the case of $\mathrm{Au}(110) / \mathrm{GaAs}(001)$ sample, which is characterized by a larger lattice mismatch and therefore by more accentuated relaxation effects. However, the BEEM spectra shown in Figs. 2(a) and 2(b) are spatial averages over $200 \times 200 \mathrm{~nm}^{2}$ areas (see below). In this way we could ob- tain spectroscopic data which are independent of the sampled area surface. We can therefore exclude a significant contribution of interfacial point defects to our data. We even notice in this context that an edge dislocation is visible in the middle of the HAADF-STEM image of the Au(110)/ GaAs(001) interface [Fig. 1(b)]. Nevertheless a nice structural continuity between atomic rows above and below the interface is still observed aside from the defect.

Figures 2(a) and 2(b) display representative BEEM spectra on $\mathrm{Au}(110) / \mathrm{GaAs}(001)$ and $\mathrm{Fe}(001) / \mathrm{GaAs}(001)$, respectively. The collector BEEM current $I_{\mathrm{BEEM}}$ versus electron energy $E$ was recorded while keeping the STM feedback loop on so as to maintain the injected tunnel current $I_{\mathrm{T}}$ constant. $I_{\mathrm{BEEM}}(E)$ curve are, respectively, a spatial average of 6400 spectra recorded on a $200 \times 200 \mathrm{~nm}^{2}$ area for $\mathrm{Au}(110) /$ $\mathrm{GaAs}(001)$ and a spatial average of 400 spectra recorded on a $200 \times 200 \mathrm{~nm}^{2}$ area for $\mathrm{Fe}(001) / \mathrm{GaAs}(001)$. Reproducible data were acquired at different locations on the sample as well as on different samples. We notice that the high tunnel current $\left(I_{\mathrm{T}}=10 \mathrm{nA}\right.$ for Au films and $I_{\mathrm{T}}=20 \mathrm{nA}$ for Fe films) used during acquisitions was necessary to obtain sufficient signal-to-noise ratio for the BEEM spectroscopic data. As a consequence, tip-surface material exchanges were often observed on metal films while sweeping the tunnel voltage to high values during BEEM spectra recording. We thus generally used $\mathrm{W}$ tips with larger tip radius to achieve improved tip stability during BEEM measurements. Those tips presented slightly lower lateral resolution than the sharpest tips used to obtain the atomically resolved STM images presented in Fig. 1.

Both BEEM spectra were fitted by using a LudekePrietsch (LP) 5/2 power law: ${ }^{2} I_{\mathrm{BEEM}}=I_{\mathrm{T}} \sum_{i=\Gamma, L, X} a_{i}\left(E-\Phi_{i}\right)^{5 / 2}$, with $\Phi_{\Gamma, L, X}$ the threshold energies corresponding to the injection of hot electrons in the $\Gamma, L$, and $X$ valleys of GaAs conduction band, respectively. ${ }^{1,3,14}$ Notice that the magnitude difference in the BEEM currents measured at the $\mathrm{Au}(110)$ / $\mathrm{GaAs}(001)$ and $\mathrm{Fe}(001) / \mathrm{GaAs}(001)$ interfaces, which can be expressed by the two $\Gamma$ amplitudes, $a_{\Gamma, \mathrm{Au}}=25.00 \times 10^{-3}$ and $a_{\Gamma, \mathrm{Fe}}=0.48 \times 10^{-3}$, can be qualitatively explained by the combined effect of: (a) the large difference in the hotelectron attenuation length in $\mathrm{Au}(15 \mathrm{~nm})$ and $\mathrm{Fe}(3 \mathrm{~nm})$, which brings almost a factor of 4 less for the iron-based film; and (b) the broader transverse momentum distribution in the $\mathrm{Fe}(001)$ film around the $\Gamma$ valley of GaAs (see Fig. 4), which brings a factor of about ten less for the case of the iron-based film. The residual difference could be caused by the different transmission matrix elements at the two interfaces.

In the case of $\mathrm{Au}(110) / \mathrm{GaAs}(001)$, the mean value of $\Phi_{\Gamma}=0.81 \pm 0.04 \mathrm{eV}$ corresponds to the local SBH at the $\mathrm{Au}(110) / \mathrm{GaAs}(001)$ interface, in good agreement with macroscopic diffusive transport measurements. The $\Phi_{L}$ $=1.14 \pm 0.04 \mathrm{eV}$ threshold energy agrees well with the position of the $L$ valley deduced from theoretical calculations of the GaAs band structure. ${ }^{15,16}$ Interestingly, the spectroscopic weight related to the $L$ valley, $a_{L}$, is in average about five times larger than $a_{\Gamma}$, a feature which underlines the strong injection of electrons with large transverse momentum at the epitaxial $\mathrm{Au}(110) / \mathrm{GaAs}(001)$ interface. In comparison, electron transport through the $\mathrm{Fe}(001) / \mathrm{GaAs}(001)$ contact [Fig. $2(\mathrm{~b})]$ can be accurately fitted by introducing just one contri- 
bution, the one of the GaAs $\Gamma$ valley. The mean value of $\Phi_{\Gamma}=0.76 \pm 0.04 \mathrm{eV}$ is again in good agreement with macroscopic diffusive transport measurements. Remarkably, we got zero spectral weight for the $L$ valley contribution, a feature to be discussed below. We shall not discuss here the higher-energy data, corresponding to electron injection in the $X$ valley of GaAs conduction band. Their energy position is correctly recovered compared to band-structure calculations $^{15,16}$ (e.g., $\Phi_{X}=1.27 \pm 0.04 \mathrm{eV}$ for $\mathrm{Au} / \mathrm{GaAs}$ ), but their spectral weights $a_{X}$ are too fluctuating, with a statistical error compatible with their absence, which does not allow an accurate determination in neither system.

The main question arising from our data is which selection rule makes the GaAs $L$ valley contribution to disappear in the case of $\mathrm{Fe} / \mathrm{GaAs}$. One might have argued that the different surface roughness of the $\mathrm{Fe} / \mathrm{GaAs}$ and $\mathrm{Au} / \mathrm{GaAs}$ samples could have been responsible of the differences found in the BEEM spectra. It is true that the different terraces size (typically $85 \times 50 \mathrm{~nm}^{2}$ for $\mathrm{Au}$ and $5 \times 2 \mathrm{~nm}^{2}$ for $\mathrm{Fe}$ ) and surface roughness can increase the transverse momentum distribution of the hot-electron beam during the tip-surface tunneling process (see, e.g., Fig. 3 of Ref. 8). However, in the case of $\mathrm{Fe} / \mathrm{GaAs}$, this at most would have led to a further contribution at the $L$ valley, and not to its disappearance, as experimentally detected. Our answer, as we shall infer below, is that the selection rule comes from the different band structure of the two thin films: the first $(\mathrm{Au})$ allows a direct injection of electrons into the $L$ valley, whereas the second $(\mathrm{Fe})$ does not.

Our theoretical analysis is based on the idea of Ref. 7 with one major simplification: we do not use the full out-ofequilibrium Keldysh formalism, but just the equilibrium electronic structure of $\mathrm{Au}, \mathrm{Fe}$, and GaAs. This approach was used already in Ref. 17 with a good semiquantitative agreement. The metal band-structure calculations are performed with a tight-binding (TB) procedure. TB parameters were obtained, from Ref. 18, through scalar relativistic selfconsistent augmented plane-wave calculations, with a 20 $k$-point mesh in the irreducible Brillouin zone and the HedinLunqvist prescription ${ }^{19}$ for the exchange-correlation potential. We could therefore calculate the equipotential energy surfaces in the metal layer, whose normals provide, as a function of $\vec{k}$, the allowed directions of propagation for the electron, and whose curvature represents the weight of each of these directions. ${ }^{17}$ As an exemplification of the method we show, in Fig. 3, the case of $\mathrm{Fe}$ (that of Au can be deduced by inspection of Fig. 1 in Ref. 7). We cut the Brillouin zone of Fe along the two planes $\Gamma N P H$ and $\Gamma H N H(N \equiv[110], H$ $\equiv[001], P \equiv[111])$. The light-colored surfaces identify the $\vec{k}$-space regions that provide the main current contribution reaching the GaAs interface in the experimentally available energy range. We then consider, as a function of the energy, the overlap of the bidimensional projection of these regions along the $[001]_{\mathrm{Fe}}$ direction with the bidimensional empty density of states in GaAs projected along the $[001]_{\mathrm{GaAs}}$ direction. GaAs density of states was calculated from the electronic band structure obtained through the ABINIT code. ${ }^{20} \mathrm{We}$ also performed the same analysis for the $\mathrm{Au}(110) / \mathrm{GaAs}(001)$ system. The bidimensional Brillouin zones of the two inter-
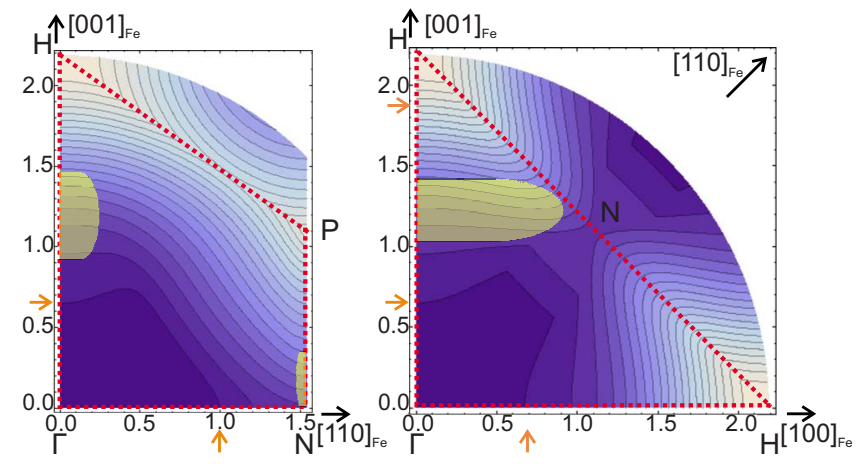

FIG. 3. (Color online) Cut of the first Brillouin zone of bce Fe in the two planes $\Gamma N P H$ and $\Gamma H N H$ (dashed lines). Continuous lines represent equipotential energy contours and small arrows show the Fermi energy. The separation between each energy contour is 0.49 $\mathrm{eV}$. Light-gray surfaces identify the $\vec{k}$-space regions that provide the main current contribution reaching the GaAs interface almost perpendicularly.

faces are represented in Figs. 4(a) and 4(b). In both figures, the black continuous circle represents the extension in the $k_{\|}$ plane of the available density of states at GaAs $\Gamma$ valley. The dashed black ellipses represents the $k_{\|}$projected available density of states at GaAs $L$ valley. The gray lines enclose the $\vec{k}$ surfaces where electron injection from Au [Fig. 4(a)] or Fe [Fig. 4(b)] is possible. Conservation of $k_{\|}$implies that a current crosses the interface only if, for a given energy, points of

(a)

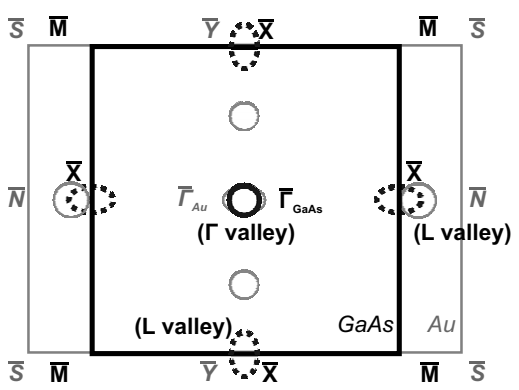

(b)

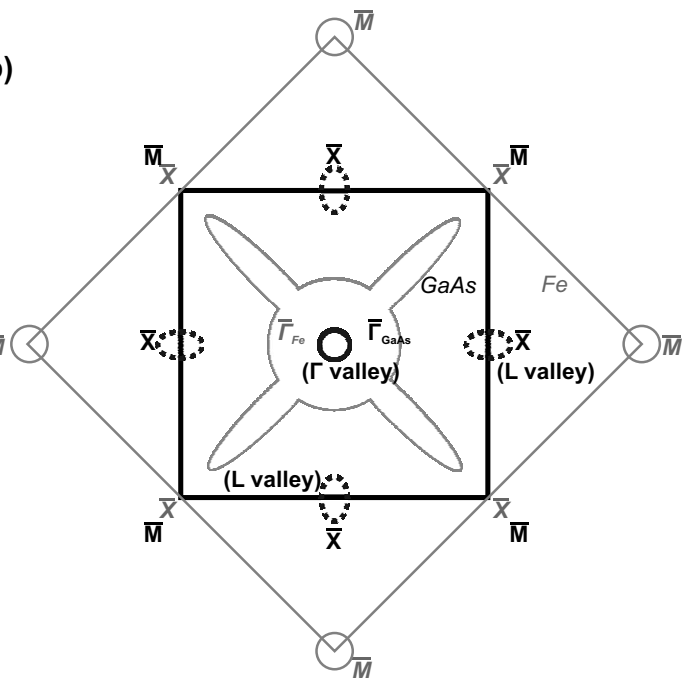

FIG. 4. Two-dimensional Brillouin zones of (a) $\mathrm{Au}(110) /$ $\mathrm{GaAs}(001)$ system and (b) $\mathrm{Fe}(001) / \mathrm{GaAs}(001)$ system. See text for more details. 
electron impinging from the metal film have a correspondent empty density of states in GaAs with same $k_{\|}$. From these projections it is clear that the band structure of iron does not allow direct electron injection in the semiconductor at $\vec{k}$ points corresponding to the empty density of states of GaAs $L$ valley. In fact, there is no overlap between the corresponding surfaces and therefore there is no transmission at this energy for the $\mathrm{Fe}(001) / \mathrm{GaAs}(001)$ interface. The opposite situation happens for the $\mathrm{Au}(110) / \mathrm{GaAs}(001)$ interface where such a contribution is present (along the $\overline{N \Gamma N}$ direction) and it is the one providing the spectral weight for the $L$ valley.

In conclusion, we have shown that ballistic electron transport through high-quality epitaxial Schottky contacts is determined by coupled metal-semiconductor band-structure characteristics. A comparative investigation of the two model interfaces, $\mathrm{Au}(110) / \mathrm{GaAs}(001)$ and $\mathrm{Fe}(001) / \mathrm{GaAs}(001)$ points out that the absence of propagating electronic states in $\mathrm{Fe}$, at the energy and transverse momentum corresponding to the GaAs $L$ valley, is responsible for major line-shape modifications of BEEM spectra. A simple theoretical analysis ${ }^{7}$ assuming coherent transport in the metal periodic potential and transverse momentum conservation at the MS interface accounts for the experimental observations. These general results suggest that the $\mathrm{GaAs}(001)$ substrate can be used in BEEM experiments as an angle-resolved analyzer of the electronic propagating states above Fermi level in epitaxial metallic films.

We thank Regis Ravelle-Chapuis and the JEOL Co. for providing us with the HAADF-STEM measurements. Financial support from Rennes Metropole and Region Bretagne is gratefully acknowledged.
${ }^{1}$ W. J. Kaiser and L. D. Bell, Phys. Rev. Lett. 60, 1406 (1988); L. D. Bell and W. J. Kaiser, ibid. 61, 2368 (1988).

${ }^{2}$ M. Prietsch, Phys. Rep. 253, 163 (1995).

${ }^{3}$ V. Narayanamurti and M. Kozhevnikov, Phys. Rep. 349, 447 (2001).

${ }^{4}$ M. K. Weilmeier, W. H. Rippard, and R. A. Buhrman, Phys. Rev. B 59, R2521 (1999).

${ }^{5}$ L. J. Schowalter and E. Y. Lee, Phys. Rev. B 43, 9308 (1991); D. L. Smith, E. Y. Lee, and V. Narayanamurti, Phys. Rev. Lett. 80, 2433 (1998).

${ }^{6}$ R. Ludeke and A. Bauer, Phys. Rev. Lett. 71, 1760 (1993).

${ }^{7}$ F. J. García-Vidal, P. L. de Andres, and F. Flores, Phys. Rev. Lett. 76, 807 (1996); P. L. de Andres, F. J. García-Vidal, K. Reuter, and F. Flores, Prog. Surf. Sci. 66, 3 (2001).

${ }^{8}$ C. A. Bobisch, A. Bannani, Y. M. Koroteev, G. Bihlmayer, E. V. Chulkov, and R. Moller, Phys. Rev. Lett. 102, 136807 (2009).

${ }^{9}$ S. Guézo, P. Turban, C. Lallaizon, J. C. Le Breton, P. Schieffer, B. Lépine, and G. Jézéquel, Appl. Phys. Lett. 93, 172116 (2008).

${ }^{10}$ S. Guézo, P. Turban, G. Delhaye, B. Lépine, P. Schieffer, and D. Sébilleau (unpublished).

${ }^{11}$ P. Schieffer, A. Guivarc'h, C. Lallaizon, B. Lépine, D. Sébilleau, P. Turban, and G. Jézéquel, Appl. Phys. Lett. 89, 161923 (2006).
${ }^{12}$ J. Herfort, W. Braun, A. Trampert, H.-P. Schönherr, and K. H. Ploog, Appl. Surf. Sci. 237, 181 (2004).

${ }^{13}$ O. Thomas, Q. Shen, P. Schieffer, N. Tournerie, and B. Lépine, Phys. Rev. Lett. 90, 017205 (2003).

${ }^{14}$ D. L. Smith, M. Kozhevnikov, E. Y. Lee, and V. Narayanamurti, Phys. Rev. B 61, 13914 (2000).

${ }^{15}$ J. S. Blakemore, J. Appl. Phys. 53, R123 (1982).

${ }^{16} \mathrm{~K}$. Hess, Advanced Theory of Semiconductor Devices (IEEE, New York, 1999).

${ }^{17}$ K. Reuter, P. L. de Andres, F. J. Garcia-Vidal, D. Sestovic, F. Flores, and K. Heinz, Phys. Rev. B 58, 14036 (1998).

${ }^{18}$ D. A. Papaconstantopoulos, Handbook of the Band Structure of Elemental Solids (Plenum, New York, 1986).

${ }^{19}$ L. Hedin and B. I. Lundqvist, J. Phys. C 4, 2064 (1971).

${ }^{20}$ X. Gonze, J.-M. Beuken, R. Caracas, F. Detraux, M. Fuchs, G.-M. Rignanese, L. Sindic, M. Verstraete, G. Zerah, F. Jollet, M. Torrent, A. Roy, M. Mikami, Ph. Ghosez, J.-Y. Raty, and D. C. Allan, Comput. Mater. Sci. 25, 478 (2002); X. Gonze, G.-M. Rignanese, M. Verstraete, J.-M. Beuken, Y. Pouillon, R. Caracas, F. Jollet, M. Torrent, G. Zerah, M. Mikami, Ph. Ghosez, M. Veithen, J.-Y. Raty, V. Olevano, F. Bruneval, L. Reining, R. Godby, G. Onida, D. R. Hamann, and D. C. Allan, Z. Kristallogr. 220, 558 (2005). 